\title{
PRIME IDEALS IN NONASSOCIATIVE RINGS
}

\author{
BY \\ BAILEY BROWN AND NEAL H. MCCOY
}

1. Introduction. Throughout this paper we shall find it convenient to use the word ring in the sense of not-necessarily-associative ring. A ring in the usual sense, that is, a ring in which multiplication is assumed to be associative, may be referred to as an associative ring.

An ideal $P$ in the arbitrary ring $R$ is said to be a prime ideal if $A B \subseteq P$, where $A$ and $B$ are ideals in $R$, implies that $A \subseteq P$ or $B \subseteq P$. In this definition it does not matter whether $A B$ is defined to be the set of all finite sums $\sum a_{i} b_{i}\left(a_{i} \in A, b_{i} \in B\right)$, or the least ideal of $R$ which contains all products $a_{i} b_{i}$, or merely the set of all these products. Behrens [4] has used the second of these definitions and Amitsur [1] the third. Throughout the present paper, if $A$ and $B$ are ideals or, more generally, any sets of elements of a ring $R$, by $A B$ we shall mean the set of all elements of $R$ of the form $a b$, where $a \in A$ and $b \in B$.

The purpose of this paper is to introduce and study certain classes of prime ideals in an arbitrary ring. Before summarizing our results, it will be necessary to introduce an appropriate notation.

Let $x_{1}=x, x_{2}, \cdots$ be a denumerable set of indeterminates which we may use to form nonassociative products in a formal way. Henceforth we let $\mathfrak{A}$ denote the set of all these indeterminates together with all finite formal products of these indeterminates in any association. If $u \in \mathfrak{A}$ and $u$ does not contain $x_{n+1}, x_{n+2}, \cdots$, we may write $u\left(x_{1}, x_{2}, \cdots, x_{n}\right)$. If $u\left(x_{1}, x_{2}, \cdots, x_{n}\right)$ $\in \mathfrak{A}$, then $u(x, x, \cdots, x)$ is a well-defined element of $\mathfrak{A}$ which we may denote by $u^{*}(x)$. For example, if $u\left(x_{1}, x_{2}, x_{3}\right)=\left(\left(x_{2} x_{1}\right) x_{3}\right) x_{1}$, then $u^{*}(x)=((x x) x) x$. We henceforth denote by $\mathfrak{B}$ the set of all elements of $\mathfrak{A}$ which do not contain $x_{2}, x_{3}, \ldots$; that is, an element of $\mathfrak{B}$ is either $x$ or some product of $x$ with itself. It follows that if $u\left(x_{1}, x_{2}, \cdots, x_{n}\right) \in \mathfrak{A}$, the mapping $u\left(x_{1}, x_{2}, \cdots, x_{n}\right)$ $\rightarrow u(x, x, \cdots, x)=u^{*}(x)$ is a mapping of $\mathfrak{A}$ onto $\mathfrak{B}$.

Now let $u\left(x_{1}, x_{2}, \cdots, x_{n}\right)$ be a fixed element of $\mathfrak{A}$. An ideal $P$ in $R$ may be said to be $u$-prime if $u\left(A_{1}, A_{2}, \cdots, A_{n}\right) \subseteq P$ implies that some $A_{i} \subseteq P$, where the $A_{i}$ are ideals in $R$. In the special case in which $u=x_{1} x_{2}$, a $u$-prime ideal is just a prime ideal and a $u^{*}$-prime ideal is a semi-prime ideal. In any ring and for any $u \in \mathfrak{A}$ which contains at least two different indeterminates, a $u$-prime ideal is necessarily prime, and a $u^{*}$-prime ideal is semi-prime. However, the converses need not be true, as examples given in the next section will show. In an associative ring the concepts of prime and $u$-prime coincide for any such $u$, as do also the concepts of semi-prime and $u^{*}$-prime.

In analogy with the $m$-systems introduced in [7], we shall call a subset

Presented to the Society, December 28, 1956; received by the editors February 1, 1957. 
$M$ of $R$ a $u$-system if whenever $A_{i}(i=1,2, \cdots, n)$ are ideals of $R$, each of which meets $M$, then $u\left(A_{1}, A_{2}, \cdots, A_{n}\right)$ meets $M$. If $A$ is an ideal in $R$, the $u$-radical $A^{u}$ of $A$ is the set of all elements $r$ of $R$ with the property that every $u$-system which contains $r$ meets $A$. We shall prove that $A^{u}$ is the intersection of all $u$-prime ideals which contain $A$. This, of course, generalizes the corresponding theorem for the associative case which was established in [7]. The special case for prime ideals $\left(u=x_{1} x_{2}\right)$ in a general ring has also been proved by Amitsur [1] and by Behrens [4].

In $\S 3$ we shall show that always $A^{u}=A^{u^{*}}$. In case $u=x_{1} x_{2}$, this reduces to a result of Amitsur [1]. Of course, in an associative ring this specializes to the well-known theorem of Levitzki [6] and Nagata [8] which states that the lower radical of Baer [2] coincides with the prime radical. Our method of proof is an adaptation of that of Nagata.

The $u$-radical of the zero ideal may naturally be called the $u$-radical of the ring $R$. This concept is discussed in $\$ 4$ where it is indicated that several of the expected properties of a radical hold for the $u$-radical.

Corresponding to each element $v$ of $\mathfrak{B}$, there is an appropriate concept of $v$-nilpotence, and the sum of all $v$-nil ideals is a greatest $v$-nil ideal. These concepts will be presented in $\$ 5$.

The radical defined by Jacobson for an associative ring has been generalized by Brown [5] to the nonassociative case. If $J$ is this radical of the ring $R$, we show in $\S 6$ that $J$ is $v$-prime for each $v \in \mathbb{B}$ and, more precisely, that a primitive ideal is itself $u$-prime for each $u \in \mathscr{A}$. In the final section we briefly indicate the relation of the results of this paper to a radical studied by Smiley [9].

2. The $u$-prime ideals and the $u$-radical of an ideal. In this section we let $u=u\left(x_{1}, x_{2}, \cdots, x_{n}\right)$ be a fixed but arbitrary element of $\mathfrak{A}$. We define the degree of $u$ in the obvious way, and we shall assume that the degree of $u$ is greater than one, that is, that $u$ is not just one of the indeterminates $x_{i}$. The integer $n$ may be any positive integer. If $P$ is an ideal in $R$, we shall use $C(P)$ to denote the complement of $P$ in $R$. If $a \in R$, the ideal in $R$ generated by $a$ will be denoted by $(a)$.

Definition 1 . An ideal $P$ in $R$ is said to be $u$-prime if it satisfies any one (and hence all three) of the following equivalent conditions:

(i) If $A_{i}(i=1,2, \cdots, n)$ are ideals in $R$ such that $u\left(A_{1}, A_{2}, \cdots, A_{n}\right)$ $\subseteq P$, then some $A_{i} \subseteq P$.

(ii) If $A_{i}(i=1,2, \cdots, n)$ are ideals in $R$, each of which meets $C(P)$, then $u\left(A_{1}, A_{2}, \cdots, A_{n}\right)$ meets $C(P)$.

(iii) If $a_{i} \in C(P)(i=1,2, \cdots, n)$, then $u\left(\left(a_{1}\right),\left(a_{2}\right), \cdots,\left(a_{n}\right)\right)$ meets $C(P)$.

Definition 2. A subset $M$ of $R$ is a $u$-system if it has one (and hence both) of the following equivalent properties:

(i) If $A_{i}(i=1,2, \cdots, n)$ are ideals of $R$, each of which meets $M$, then $u\left(A_{1}, A_{2}, \cdots, A_{n}\right)$ meets $M$. 
(ii) If $a_{i} \in M(i=1,2, \cdots, n)$, then $u\left(\left(a_{1}\right),\left(a_{2}\right), \cdots,\left(a_{n}\right)\right)$ meets $M$.

Clearly an ideal is $u$-prime if and only if its complement is a $u$-system.

Definition 3. If $A$ is an ideal in $R$, the $u$-radical $A^{u}$ of $A$ is the set of all elements $r$ of $R$ such that every $u$-system which contains $r$ meets $A$.

We may now prove the following theorem.

THEOREM 1. If $A$ is an ideal in $R, A^{u}$ is the intersection of all $u$-prime ideals which contain $A$.

Let us denote by $X$ the intersection of all $u$-prime ideals which contain $A$, and show that $A^{u}=X$.

First, we verify that $A^{u} \subseteq X$. If $P$ is a $u$-prime ideal such that $A \subseteq P$ and $b \in A^{u}$, then $C(P)$ is a $u$-system which does not meet $A$, and hence $b \notin C(P)$. That is, $b \in P$, and hence $A^{u} \subseteq P$. It follows that $A^{u} \subseteq X$, as we wished to show.

Next we show that $X \subseteq A^{u}$. Suppose that $c \notin A^{u}$. Then there exists a $u$ system $M$ which contains $c$ and does not meet $A$. By Zorn's Lemma, there exists an ideal $P$ maximal in the class of ideals which contain $A$ and do not meet $M$. We prove as follows that $P$ is $u$-prime. Suppose that $A_{i}$ $(i=1,2, \cdots, n)$ are ideals, each of which meets $C(P)$. The maximal property of $P$ implies that each of the ideals $P+A_{i}$ meets $M$. By Definition 2(i) it follows that $u\left(P+A_{1}, P+A_{2}, \cdots, P+A_{n}\right)$ meets $M$. But clearly

$$
u\left(P+A_{1}, P+A_{2}, \cdots, P+A_{n}\right) \subseteq P+u\left(A_{1}, A_{2}, \cdots, A_{n}\right) .
$$

Since $P$ does not meet $M, u\left(A_{1}, A_{2}, \cdots, A_{n}\right) \Phi P$ and hence $u\left(A_{1}, A_{2}, \cdots, A_{n}\right)$ meets $C(P)$. By Definition 1(ii), we see that $P$ is a $u$-prime ideal. Now since $c \notin P, c \notin X$, and it follows that $X \subseteq A^{u}$, completing the proof.

REMARK. Let us write $u_{1}<u_{2}$ if $u_{1}$ and $u_{2}$ are distinct elements of $\mathfrak{A}$ such that $u_{1}$ is contained as a factor in $u_{2}$. That is, $u_{2}$ is a product of $u_{1}$ and certain of the indeterminates $x_{i}$ in some association. For example, $u_{1}<u_{2}$ if $u_{1}$ $=\left(x_{1} x_{2}\right) x_{3}$ and $u_{2}=x_{1}\left(\left(\left(x_{1} x_{2}\right) x_{3}\right) x_{2}\right)$. If $u_{1}<u_{2}$, then an ideal which is $u_{2}$-prime is also $u_{1}$-prime; hence $A^{u_{1}} \subseteq A^{u_{2}}$. Under what conditions this inclusion will be proper is an unsolved problem. The examples which we now give will shed a little light on this, and also illustrate the concept of $u$-prime ideal.

EXAMPLE 1. Let $R$ be the algebra over an arbitrary field $F$, with basis elements $z_{0}, z_{1}, z_{2}, z_{i}$, having the following multiplication table.

\begin{tabular}{|c|c|c|c|c|}
\hline & $z_{0}$ & $z_{1}$ & $z_{2}$ & $z_{3}$ \\
\hline$z_{0}$ & $z_{0}$ & $z_{1}$ & $z_{2}$ & $z_{3}$ \\
\hline$z_{1}$ & $z_{1}$ & 0 & 0 & $z_{2}$ \\
\hline$z_{2}$ & $z_{2}$ & 0 & $z_{3}$ & $z_{3}$ \\
\hline$z_{3}$ & $z_{3}$ & $z_{2}$ & 0 & 0 \\
\hline
\end{tabular}


Clearly $z_{0}$ is the unit element of $R$. If $a_{i} \in R(i=1,2, \cdots, n)$, we denote by $\left[a_{1}, a_{2}, \cdots, a_{n}\right]$ the set of all linear combinations $\sum \alpha_{i} a_{i}, \alpha_{i} \in F$; hence we may write $R=\left[z_{0}, z_{1}, z_{2}, z_{3}\right]$. It is easy to verify that the only proper ideals of $R$ are $M=\left[z_{1}, z_{2}, z_{3}\right]$ and $N=\left[z_{2}, z_{3}\right]$. Now $N^{2}=\left[z_{3}\right], N N^{2}=\left[z_{3}\right]$, and $N^{2} N=0$. If we set $u_{1}=\left(x_{1} x_{2}\right) x_{3}$ and $u_{2}=x_{1}\left(x_{2} x_{3}\right)$, by using the fact that $N$ is contained in every nonzero ideal of $R$, it follows that the zero ideal is prime and also $u_{2}$-prime, but is not $u_{1}$-prime.

EXAmple 2. Let $R$ be the algebra over a field $F$, with basis elements $z_{0}, z_{1}, \cdots, z_{n}(n>3)$ whose multiplication is defined as follows. The multiplication is assumed to be commutative, $z_{0}$ is the unit element of $R$,

$$
z_{1} z_{2}=z_{3}, z_{1} z_{n}=z_{3} ; z_{3}^{2}=z_{4}, z_{4}^{2}=z_{5}, \cdots, z_{n-1}^{2}=z_{n}, z_{n}^{2}=z_{2},
$$

and all other products are zero. It is easy to verify that the linear sets $M$ $=\left[z_{1}, z_{2}, \cdots, z_{n}\right]$ and $N=\left[z_{2}, z_{3}, \cdots, z_{n}\right]$ are ideals in $R$. We proceed to verify that these are the only proper ideals.

If $r=\alpha_{0} z_{0}+\alpha_{1} z_{1}+\cdots+\alpha_{n} z_{n}$ is an element of $R$ with $\alpha_{0} \neq 0$, we show that the ideal $(r)$ generated by $r$ is $R$ itself. We have $\left(r z_{2}\right) z_{1}=\alpha_{0} z_{3}$ and, since $R$ has $z_{0}$ as unit element, it follows that $z_{3} \in(r)$. Then from the multiplication table, we see in turn that $z_{4}, \cdots, z_{n}, z_{2}$ are in $(r)$. Since $r \in(r)$, it follows that $\alpha_{0} z_{0}+\alpha_{1} z_{1} \in(r)$ and hence $z_{1}\left(\alpha_{0} z_{0}+\alpha_{1} z_{1}\right)=\alpha_{0} z_{1} \in(r)$ and $z_{1} \in(r)$. Finally, then, $z_{0} \in(r)$ and $(r)=R$.

Now let $s=\beta_{1} z_{1}+\cdots+\beta_{n} z_{n}$ be an element of $R$ with $\beta_{1} \neq 0$. Then $s z_{2}=\beta_{1} z_{3}$ and $z_{3} \in(s)$. In turn, $z_{4}, \cdots, z_{n}, z_{2}$ are in $(s)$, and finally $z_{1} \in(s)$. Hence $(s)=M$.

We now let $t=\gamma_{2} z_{2}+\cdots+\gamma_{n} z_{n}$, where some one of the $\gamma_{i} \neq 0$, and show that $(t)=N$. If $\gamma_{n} \neq 0, t z_{n}=\gamma_{n} z_{2}$ implies that $z_{2} \in(t)$, and it follows easily that $(t)=N$. Suppose then that $\gamma_{n}=0, \gamma_{2} \neq 0$. Then $t z_{1}=\gamma_{2} z_{3}$ and again $(t)=N$. If $\gamma_{2}=\gamma_{n}=0$ but $\gamma_{i} \neq 0$ for some $i(2<i<n)$, the same conclusion is easily obtained. This shows that $M$ and $N$ are the only proper ideals in $R$.

If we define $N^{(1)}=N$, and generally $N^{(k)}=N^{(k-1)} N$, we find that $N^{(2)}$ $=\left[z_{2}, z_{4}, z_{5}, \cdots, z_{n}\right], N^{(3)}=\left[z_{2}, z_{5}, \cdots, z_{n}\right], \cdots, N^{(n-1)}=\left[z_{2}\right], N^{(n)}=0$.

We now define elements of $\mathfrak{A}$ as follows. Let $u_{1}=x_{1}, u_{2}=u_{1} x_{2}, \cdots, u_{k+1}$ $=u_{k} x_{k+1}, \cdots$. Since $N$ is contained in every nonzero ideal of $R$, it follows that in $R$ the zero ideal is $u_{i}$-prime for $i<n$, but is not $u_{n}$-prime.

3. Equivalence of $u$-radical and $u^{*}$-radical. If $u=u\left(x_{1}, x_{2}, \cdots, x_{n}\right)$ is an element of $\mathfrak{A}$, we shall use the notation given in the introduction and set $u^{*}=u^{*}(x)=u(x, x, \cdots, x)$ with $x=x_{1}$. Thus $u^{*} \in \mathfrak{B} \subseteq \mathfrak{A}$, and hence the general definitions and results of the preceding section can be applied with $u$ replaced by $u^{*}$. Moreover, the examples given above show that in the nonassociative case, the concepts of prime and $u$-prime need not coincide, and neither do the concepts of semi-prime and $u^{*}$-prime.

We next establish the following theorem.

Theorem 2. If $A$ is an ideal in $R$ and $u \in \mathfrak{A}$, then $A^{u}=A^{u^{*}}$. 
It is obvious that an ideal which is $u$-prime is also $u^{*}$-prime, and hence that $A^{u^{*}} \subseteq A^{u}$. Inclusion the other way will follow easily from the following lemma.

Lemma. If $a$ is an element of $a u^{*}$-system $S$, there exists a $u$-system $M$ such that $a \in M \subseteq S$.

Let $M=\left\{a_{1}, a_{2}, \cdots\right\}$, where $a_{1}=a$ and the other elements of $M$ are defined inductively as follows. Since $a_{1} \in S$, by Definition 2(ii), $u\left(\left(a_{1}\right),\left(a_{1}\right)\right.$, $\left.\cdots,\left(a_{1}\right)\right)$ meets $S$. Let $a_{2} \in u\left(\left(a_{1}\right),\left(a_{1}\right), \cdots,\left(a_{1}\right)\right) \cap S$. Then let, in general, $a_{k} \in u\left(\left(a_{k-1},\left(a_{k-1}\right), \cdots,\left(a_{k-1}\right)\right) \cap S\right.$. Thus $M$ is defined, and it remains to show that $M$ is a $u$-system. Since, for each $k, a_{k} \in\left(a_{k-1}\right)$, it follows that if $a_{i_{1}}, a_{i_{2}}, \cdots, a_{i_{n}} \in M$ with $i_{1} \leqq i_{2} \leqq \cdots \leqq i_{n}$, then $a_{i_{n}+1} \in u\left(\left(a_{i_{n}}\right),\left(a_{i_{n}}\right), \cdots\right.$, $\left.\left(a_{i_{n}}\right)\right) \subseteq u\left(\left(a_{i_{1}}\right),\left(a_{i_{2}}\right), \cdots,\left(a_{i_{n}}\right)\right)$. This establishes the lemma.

Now to show that $A^{u} \subseteq A^{u^{*}}$, let $a \in A^{u}$ and let $S$ be a $u^{*}$-system which contains $a$. By the lemma, there exists a $u$-system $M$ such that $a \in M \subseteq S$. By the definition of $A^{u}$, it follows that $M$ meets $A$ and therefore $S$ meets $A$. Hence we have $a \in A^{u^{*}}$, and therefore $A^{u} \subseteq A^{u^{*}}$, completing the proof of the theorem.

For the special case in which $u=x_{1} x_{2}$, this theorem states that the prime radical of an ideal coincides with its lower radical. This has been established by Amitsur [1]; and if $R$ is an associative ring, it is a well-known result of Levitzki [6] and Nagata [8].

It is clear that $u^{*}$-prime ideals are closed under arbitrary intersection and hence that $A^{u^{*}}$ is the smallest $u^{*}$-prime ideal which contains $A$. We therefore have the following immediate corollary of Theorems 1 and 2.

Corollary. If $A$ is an ideal in $R$, then $A=A^{u^{*}}$ if and only if $A$ is an intersection of u-prime ideals.

4. The $u$-radical of a ring. We now make the following definition.

Definition 4. The $u$-radical of the zero ideal in a ring $R$ may be called the $u$-radical of the ring $R$.

We shall find it convenient to denote the $u$-radical of $R$ by $R^{u}$.

Definition 5. A ring $R$ is said to be a $u$-prime ring if in $R$ the zero ideal is $u$-prime.

It is now clear that if $P$ is an ideal in a ring $R$, then $R / P$ is a $u$-prime ring if and only if $P$ is a $u$-prime ideal.

Inasmuch as the proofs follow easily by the methods of [7], we state the following two theorems without proof.

THEOREM 3. A necessary and sufficient condition that a ring $R$ be isomorphic to a subdirect sum of u-prime rings is that $R^{u}=0$.

THEOREM 4. The ring $R / R^{u}$ has zero u-radical.

We next prove the following result. 
THEOREM 5. Let $R^{u}$ and $S^{u}$ be the respective $u$-radicals of the rings $R$ and $S$. If $R$ is contained in $S$ in such a way that each ideal in $R$ is also an ideal in $S$, then $R^{u}=S^{u} \cap R$.

We show first that if $P$ is a $u$-prime ideal in $S$, then $P \cap R$ is a $u$-prime ideal in $R$. Suppose that $u\left(A_{1}, A_{2}, \cdots, A_{n}\right) \subseteq P \cap R$, where the $A_{i}$ are ideals in $R$. Since $P$ is a $u$-prime ideal in $S$ and the $A_{i}$ are also ideals in $S$, it follows that some $A_{i} \subseteq P \cap R$. This shows that $P \cap R$ is a $u$-prime ideal in $R$. Theorem 1 then implies that $R^{u} \subseteq S^{u} \cap R$.

To prove inclusion the other way, let $a \in S^{u} \cap R$. Then every $u$-system in $S$ which contains $a$ contains 0 . Since a $u$-system in $R$ is also a $u$-system in $S$, it follows that every $u$-system in $R$ which contains $a$ contains 0 ; hence that $a \in R^{u}$. This completes the proof.

In the associative case the conclusion of Theorem 5 is true without the requirement that any ideal in $R$ be an ideal in $S$. However, if associativity is not assumed, there is at least one case of some interest in which this requirement is met. In any of the usual methods of imbedding a ring $R$ in a ring $S$ with unit element, the construction yields a ring $S$ such that the hypotheses of Theorem 5 are satisfied. We may therefore state the following corollary.

CoROllary. The ring $R$ may be imbedded in a ring $S$ with unit element in such a way that for every $u \in \mathfrak{A}$ we have $R^{u}=S^{u} \cap R$.

If $R$ is an arbitrary ring, we shall denote by $R_{m}$ the ring of all square matrices of order $m$ with elements in $R$. We next prove the following result.

LEMma. The ring $S$ with unit element is a u-prime ring if and only if $S_{m}$ is a u-prime ring.

First we assume that $S$ is not $u$-prime. Suppose that $u\left(\left(a_{1}\right),\left(a_{2}\right), \cdots\right.$. $\left.\left(a_{n}\right)\right)=0$ where each $a_{i}$ is a nonzero element of $S$. If $e_{i j}$ is the element of $S_{m}$ with 1 in the $(i, j)$ position and zeros elsewhere, it follows, for example, that $u\left(\left(a_{1} e_{11}\right),\left(a_{2} e_{11}\right), \cdots,\left(a_{n} e_{11}\right)\right)=0$ since each element of the left member is a matrix having in each position a sum of elements of $u\left(\left(a_{1}\right),\left(a_{2}\right), \cdots,\left(a_{n}\right)\right)$. Now each $a_{i} e_{11} \neq 0$ and we see tht $S_{m}$ is not $u$-prime.

Conversely, suppose that $S_{m}$ is not $u$-prime and that $u\left(A_{1}, A_{2}, \cdots, A_{n}\right)$ $=0$ where each $A_{i}$ is a nonzero ideal of $S_{m}$. Let $T_{i}$ be a nonzero matrix in $A_{i}$, and suppose that $T_{i}$ is the matrix having $t_{p q}^{(i)}$ in the $(p, q)$ position, with $t_{p_{i} q_{i}}^{(i)} \neq 0$. Then $e_{1 p_{i}} T_{i} e_{q_{i} 1}=t_{p_{i q} i}^{(i)} e_{11} \in A_{i}$. Moreover, if $a_{i}$ is any element of the ideal in $S$ generated by $t_{p_{i} q_{i}}^{(i)}$, we have $a_{i} e_{11} \in A_{i}$. It follows that

$$
u\left(a_{1} e_{11}, a_{2} e_{11}, \cdots, a_{n} e_{11}\right)=u\left(a_{1}, a_{2}, \cdots, a_{n}\right) e_{11}=0 .
$$

Hence $u\left(a_{1}, a_{2}, \cdots, a_{n}\right)=0$. This shows that $S$ is not $u$-prime since $a_{i}$ is an arbitrary element of the nonzero ideal in $S$ generated by $t_{p_{i} q_{i}}^{(1)}$. The lemma is therefore established.

The following theorem follows easily from this lemma by the method of 
proof of the corresponding theorem in the associative case. In the proof, our Theorem 5 above is used in place of the stronger result available in that case [7, Theorem 4].

THEOREM 6. If $R$ is any ring and $u \in \mathfrak{A}$, then $\left(R_{m}\right)^{u}=\left(R^{u}\right)_{m}$.

5. Nil ideals and $v$-nil ideals. There are available various concepts of nilpotence when multiplication is not associative. The weakest of these is the following.

Definition 6. An element $a$ of the ring $R$ is nilpotent if there exists an element $v(x)$ of $\mathfrak{B}$ such that $v(a)=0$. An ideal is a nil ideal if each of its elements is nilpotent.

This is equivalent to the definition used by Behrens [3], who proved that the sum of all nil ideals is a nil ideal and hence that there exists a unique greatest nil ideal. This ideal we may denote by $N(R)$. It is easy to see that $N(R)$ is $v$-prime for each $\| \in \mathfrak{B}$. For let $A$ be an ideal in $R$ such that $v(A)$ $\subseteq N(R)$. If $a \in A$, then in particular we have $v(a) \in N(R)$. Hence there exists $w \in \mathfrak{B}$ such that $w(v(a))=0$. This shows that $a$ is nilpotent, and it follows that $A \subseteq N(R)$, completing the proof.

We now introduce a different concept of nilpotence which bears the same relation to an arbitrary fixed element $v(x)$ of $\mathfrak{B}$ that solvability does to the element $x \boldsymbol{x}$ of $\mathfrak{B}$. Let us introduce a sequence of elements of $\mathfrak{B}$ as follows:

$$
v^{(0)}(x)=x, v^{(1)}(x)=v(x), \cdots, v^{(k+1)}(x)=v\left(v^{(k)}(x)\right), \cdots .
$$

We now make the following definition.

Definition 7. An element $a$ of $R$ is said to be $v$-nilpotent if $v^{(m)}(a)=0$ for some nonnegative integer $m$. An ideal is a $v$-nil ideal if each of its elements is $v$-nilpotent.

The next theorem will show how $v$-nilpotence is related to other concepts of this paper.

THEOREM 7. The sum of all v-nil ideals of the ring $R$ is a greatest v-nil ideal $N_{v}(R)$, and if $u\left(x_{1}, x_{2}, \cdots, x_{n}\right)$ is any element of $\mathfrak{A}$ such that $u^{*}(x)=v(x)$, then $R^{u} \subseteq N_{v}(R) \subseteq N(R)$. Moreover, $N_{v}(R)$ is a v-prime ideal.

As a first step in the proof, we show that for nonnegative integers $r$ and $s$,

$$
v^{(r)}\left(v^{(s)}(x)\right)=v^{(r+s)}(x) \text {. }
$$

If $r=0,1$, and $s$ is arbitrary, this is true by definition. Assume (1) for arbitrary $s$ and for the nonnegative integer $r-1$. Then, by use of this induction hypothesis and by definition, we have

$$
v^{(r)}\left(v^{(s)}(x)\right)=v\left(v^{(r-1)}\left(v^{(s)}(x)\right)\right)=v\left(v^{(r+s-1)}(x)\right)=v^{(r+s)}(x) .
$$

This establishes (1).

To show the existence of $N_{v}(R)$, we shall limit ourselves to proving that 
if $(a)$ and $(b)$ are $v$-nil ideals, then $(a-b)$ is a $v$-nil ideal. Any element of $(a-b)$ is of the form $c+d$, where $c \in(a)$ and $d \in(b)$. Since $c \in(a)$, there exists a nonnegative integer $s$ such that $v^{(s)}(c)=0$. But then $v^{(s)}(c+d)=v^{(s)}(c)+e$ $=e$, where $e \in(d) \subseteq(b)$. Since $(b)$ is a $v$-nil ideal, there exists a nonnegative integer $r$ such that $v^{(r)}(e)=0$. Using (1), we now have

$$
v^{(r+s)}(c+d)=v^{(r)}\left(v^{(s)}(c+d)\right)=v^{(r)}(e)=0,
$$

and $c+d$ is $v$-nil. This proves the existence of the greatest $v$-nil ideal $N_{v}(R)$.

It is obvious that $N_{v}(R) \subseteq N(R)$, so we now prove that $R^{u} \subseteq N_{v}(R)$. Since $R^{u}=R^{u^{*}}=R^{v}$ by Theorem 2, we shall show that $R^{v} \subseteq N_{v}(R)$. We show first that if $a \in R$, the set $M=\left\{v^{(k)}(a) ; k=0,1,2, \cdots\right\}$ is a $v$-system. Let $b$ $=v^{(k)}(a) \in M$. Then $v(b)=v^{(k+1)}(a) \in M$ and $v(b) \in v((b))$. Since $v((b))$ meets $M$, Definition 2(ii) shows that $M$ is a $v$-system. Suppose now that $a \in R^{v}$. Then the $v$-system $M$ contains $a$ and therefore contains the element 0 . It follows that $a$ is $v$-nilpotent and hence that $R^{v}$ is a $v$-nil ideal. Thus $R^{v} \subseteq N_{v}(R)$ and the proof is complete.

There remains only to prove that $N_{v}(R)$ is $v$-prime. If $A$ is an ideal in $R$ such that $v(A) \subseteq N_{v}(R)$ and $a \in A$, then $v(a) \in N_{v}(R)$. Hence $v(a)$ is $v$-nilpotent, that is, for some nonnegative integer $r, v^{(r)}(v(a))=0$. But by (1), this implies that $v^{(r+1)}(a)=0$ and hence that $a$ is $v$-nilpotent. This proves that $A \subseteq N_{v}(R)$, which shows that $N_{v}(R)$ is a v-prime ideal.

6 . The Jacobson radical. The definition of the Jacobson radical of an associative ring has been extended by Brown [5] to the nonassociative case. The present treatment is in terms of the right radical, which for nonassociative rings need not coincide with the left radical. If $a \in R$, we shall let $Q(a)$ be the right ideal of $R$ generated by the set of all elements $a t-t$, with $t \in R$. The element $a$ is quasi-regular if $a \in Q(a)$, and an ideal is quasi-regular if each of its elements is quasi-regular. The Jacobson radical of $R$ is the greatest quasi-regular ideal of $R$. We denote this radical by $J$ or by $J(R)$.

We proceed to give an elementary proof of the following result.

TheOREM 8. For any ring $R, J(R)$ is v-prime for each $v \in \mathfrak{B}$. Moreover, $N(R) \subseteq J(R)$.

We begin by proving two lemmas.

Lemma 1. For each $v(x) \in \mathfrak{B}$ and each $a \in R, v(a)=a-c$ for some $c \in Q(a)$.

This is obvious if $v(x)=x$ and also follows easily for $v(x)=x^{2}$ by use of the equation $a^{2}=a-[a(-a)-(-a)]$. We use induction on the degree of $v(x)$ and let $v(x)$ be an element of $\mathfrak{B}$ of degree $n>2$. We assume the truth of the lemma for all elements of $\mathfrak{B}$ of degree less than $n$. Now it is always possible to express $v(x)$ as a product of two elements of $\mathfrak{B}$, say $v(x)=v_{1}(x) v_{2}(x)$, where $v_{1}(x)$ and $v_{2}(x)$ have degrees less than $n$. By the induction hypothesis, we may write $v_{1}(a)=a-d$ and $v_{2}(a)=a-e$, with $d, e \in Q(a)$. Hence 


$$
v(a)=v_{1}(a) v_{2}(a)=(a-d)(a-e)=a^{2}-a e-d a+d e .
$$

Since $d \in Q(a)$, we have $d a \in Q(a)$ and $d e \in Q(a)$. Moreover, ae $\in Q(a)$ since $a e=(a e-e)+e$. Also, as pointed out above as a special case of the lemma, $a^{2}=a-f, f \in Q(a)$. Combining all of these, we see that $v(a)=a-c, c \in Q(a)$, and the lemma is established.

LEMMA 2. If $v(a)$ is quasi-regular, then a is quasi-regular.

By the preceding lemma, we have $v(a)=a-c$ where $c \in Q(a)$. Under the assumption that $v(a)$ is quasi-regular, we thus have $a-c \in Q(a-c)$. A lemma of Brown [5] shows that $Q(a-c) \subseteq Q(a)+(c)_{r}$, where $(c)_{r}$ is the right ideal in $R$ generated by $c$. Since $c \in Q(a)$, we have $(c)_{r} \subseteq Q(a)$, so finally $a \in Q(a)$. This establishes the lemma.

It is now easy to show that $J$ is $v$-prime for each $v \in \mathfrak{B}$. If $A$ is an ideal in $R$ such that $v(A) \subseteq J$ and $a \in A$, then $v(a) \in J$ and hence $v(a)$ is quasi-regular. By Lemma 2, $a$ is then quasi-regular. Hence $A \subseteq J$, which shows that $J$ is $v$-prime. The first statement of the theorem is therefore proved.

If $b \in N(R)$ and $a \in(b)$, there exists $v \in \mathfrak{B}$ such that $v(a)=0$. Lemma 2 then shows that $a$ is quasi-regular. Hence $(b)$ is a quasi-regular ideal and (b) $\subseteq J$. It follows that $N(R) \subseteq J$, completing the proof.

This theorem shows that $J=J^{u^{*}}$ for each $u \in \mathfrak{A}$ and, by the Corollary to Theorem 2, it follows that $J$ is an intersection of $u$-prime ideals for each $u \in \mathfrak{R}$. In what follows we shall sharpen this result, but first we introduce the necessary terminology.

Following Brown [5], we say that a right ideal $I$ in $R$ is modular if there exists an element $e$ of $R$ such that $e r-r \in I$ for all $r \in R$. A ring $R$ is primitive if it contains a modular maximal right ideal $M$ which contains no nonzero ideal of $R$. We say that an ideal $A$ of $R$ is primitive if the ring $R / A$ is primitive. Brown [5, Theorem 1] has shown that $J(R)$ is an intersection of primitive ideals, and we shall show below, as a corollary to the next theorem, that a primitive ideal is actually $u$-prime for each $u \in \mathfrak{A}$.

TheORem 9. Let $M$ be a modular maximal right ideal in the ring $R$, and $u\left(x_{1}, x_{2}, \cdots, x_{n}\right)$ an arbitrary element of $\mathfrak{A}$. If $A_{\boldsymbol{i}}(i=1,2, \cdots, n)$ are ideals in $R$ such that $u\left(A_{1}, A_{2}, \cdots, A_{n}\right) \subseteq M$, then some $A_{i} \subseteq M$.

The proof is by induction on the degree of $u$, and we begin by considering the special case in which $u=x_{1} x_{2}$. Suppose then that $A_{1}, A_{2}$ are ideals such that $A_{1} A_{2} \subseteq M$ with $A_{1} \Phi M$. Since $M$ is a maximal right ideal, we have $R=M+A_{1}$. Let $e$ be an element of $R$ such that $e r-r \in M$ for $r \in R$. Then we may write $e=m+a$, where $m \in M$ and $a \in A_{1}$. If $a_{2} \in A_{2}$, we have therefore $e a_{2}=m a_{2}+a a_{2}$. But $m a_{2} \in M$ since $m \in M$, and $a a_{2} \in M$ in view of our assumption that $A_{1} A_{2} \subseteq M$. Hence $e a_{2} \in M$ and the modularity of $M$ implies that $a_{2} \in M$. Thus $A_{2} \subseteq M$, as required.

We now return to the general case. There is no loss of generality in hence- 
forth assuming that in $u\left(x_{1}, x_{2}, \cdots, x_{n}\right)$ each of $x_{1}, x_{2}, \cdots, x_{n}$ appears exactly once. We therefore let $u\left(x_{1}, x_{2}, \cdots, x_{n}\right)$ be such a product of degree $n>2$ and, for convenience of notation, suppose that $x_{1}$ appears on the left, no matter what the association. Let us define $u^{\prime}\left(x_{2}, \cdots, x_{n}\right)$ to be the element of $\mathfrak{A}$ obtained from $u\left(x_{1}, x_{2}, \cdots, x_{n}\right)$ by erasing $x_{1}$ and any superfluous parentheses. For example, if $u=\left(x_{1} x_{2}\right)\left(x_{3} x_{4}\right)$, then $u^{\prime}=x_{2}\left(x_{3} x_{4}\right)$. We now assume the desired result for all elements of $\mathfrak{A}$ of degree less than $n$. Suppose that $u\left(A_{1}, A_{2}, \cdots, A_{n}\right) \subseteq M$ with $A_{1} \Phi M$. As above, we have $R=M+A_{1}$ and $e=m+a, m \in M, a \in A_{1}$. Let $a_{i} \in A_{i}(i=2, \cdots, n)$. Then

$$
u\left(e, a_{2}, \cdots, a_{n}\right)=u\left(m, a_{2}, \cdots, a_{n}\right)+u\left(a, a_{2}, \cdots, a_{n}\right) .
$$

But $u\left(m, a_{2}, \cdots, a_{n}\right) \in M$ since $m$ appears on the left. Moreover, $u\left(a, a_{2}, \cdots, a_{n}\right) \in M$ in view of our assumption that $u\left(A_{1}, A_{2}, \cdots, A_{n}\right) \subseteq M$. Hence $u\left(e, a_{2}, \cdots, a_{n}\right) \in M$. But since $e r-r \in M$ for $r \in R$, this implies that $u^{\prime}\left(a_{2}, \cdots, a_{n}\right) \in M$. This shows that $u^{\prime}\left(A_{2}, \cdots, A_{n}\right) \subseteq M$, and since $u^{\prime}$ has degree $n-1$, our induction hypothesis shows that some $A_{i} \subseteq M$ $(i=2, \cdots, n)$, and the proof is completed.

If $R$ is a primitive ring, there exists in $R$ a modular maximal right ideal $M$ which contains no nonzero ideal of $R$. Hence, in this case, we can be sure that $u\left(A_{1}, A_{2}, \cdots, A_{n}\right)=0$ implies that some $A_{i}=0$. This shows that a primitive ring is $u$-prime for each $u \in \mathfrak{A}$. Moreover, since an ideal $B$ is a $u$ prime ideal if and only if $R / B$ is a $u$-prime ring, we have the following result.

Corollary. A primitive ideal is $u$-prime for each $u \in \mathfrak{A}$.

Of course, it follows at once from this result that $J$ is $v$-prime for each $v \in \mathfrak{B}$, and this is another proof of the first statement of Theorem 8 .

Added in proof. San Soucie [10] has proved that a primitive ring is a prime ring. In particular, the special case of the preceding corollary in which $u=x_{1} x_{2}$ follows immediately from this result.

7. Relation to a radical of Smiley. Let us say that an ideal $I$ is modular if the ring $R / I$ has a unit element. It is then easy to verify that the proof of Theorem 9 carries through if $M$ is a modular maximal ideal. Hence a modular maximal ideal is $u$-prime for every $u \in \mathfrak{A}$. Now Smiley [9] has studied a radical $S(R)$ of a ring $R$, which coincides with the intersection of all modular maximal ideals. It follows that $S(R)$ is an intersection of $u$-prime ideals, and hence is $v$-prime for every $v \in \mathfrak{B}$. By the definition of $S(R)$ given in [9], it is clear that $J(R) \subseteq S(R)$. Combining this with other results in this paper, we have, for each ring $R$ and for each $u \in \mathfrak{R}$,

$$
R^{u}=R^{u^{*}} \subseteq N_{u^{*}}(R) \subseteq N(R) \subseteq J(R) \subseteq S(R) .
$$

Furthermore, $R^{u}$ and $N_{u^{*}}(R)$ are $u^{*}$-prime; while $N(R), J(R)$ and $S(R)$ are $v$-prime for every $v \in \mathfrak{B}$. 


\section{BIBLIOGRAPHY} 136.

1. S. A. Amitsur, A general theory of radicals III, Amer. J. Math. vol. 76 (1954) pp. 126-

2. R. Baer, Radical ideals, Amer. J. Math. vol. 65 (1943) pp. 537-568.

3. E. A. Behrens, Nichtassoziative Ringe, Math. Ann. vol. 127 (1954) pp. 441-452.

4. - Z Zur additiven Idealtheorie in nichtassoziativen Ringen, Math. Z. vol. 64 (1956) pp. $169-182$.

5. B. Brown, An extension of the Jacobson radical, Proc. Amer. Math. Soc. vol. 2 (1951) pp. 114-117.

6. J. Levitzki, Prime ideals and the lower radical, Amer. J. Math. vol. 73 (1951) pp. 25-29.

7. N. H. McCoy, Prime ideals in general rings, Amer. J. Math. vol. 71 (1949) pp. 823-833. 344.

8. M. Nagata, On the theory of radicals in a ring, J. Math. Soc. Japan vol. 3 (1951) pp. 330-

9. M. F. Smiley, Application of a radical of Brown and McCoy to non-associative rings, Amer. J. Math. vol. 72 (1950) pp. 93-100.

10. Added in proof. R. L. San Soucie, Weakly standard rings, Amer. J. Math. vol. 79 (1957) pp. 80-86.

Amherst College,

AMHerst, Mass.

Smith College,

Northampton, MASS. 